\section{Discussion}

The findings of lower overall cognitive performance in boys, in children from working class families, and in children of mothers who themselves had been relatively poor achievers educationally are not new and bear out the reliability of the McCarthy tests in 4 year olds. ${ }^{26}$ What is new is the selective correlation between maternal depression early in the child's life and subsequent poorer cognitive performance. The longitudinal design of the study ensured that the measures in the children were independent of the assessments of the mothers, and the cognitive tests were not subject to bias, such as may occur when mothers report behavioural problems in their children.

Causal links cannot automatically be inferred from correlations, but the independence of the association between maternal postnatal depression and children's lower cognitive performance from the sex of the child and the mother's educational achievement, and its interdependence with marital problems, father's psychiatric history, and parental social class, point to ways in which maternal postnata depression might exert its impact. The sample we studied comprised first born children, who may be particularly sensitive to impaired relationships with and between their parents.

About one in every 10 women becomes clinically depressed in the months after childbirth, ${ }^{11}{ }^{15}{ }_{28}$ and the size of the problem alone therefore merits attention. Our findings of possible deleterious effects of postnatal depression on the cognitive development of young children require replication and more extensive follow up.

This research was supported by a grant from the Mental Health Foundation. We are grateful to the families who participated in the survey and to Ms Valerie Scott, Mr P Conlon, and Mr C Sharp for help with analysis of the data.

\section{References}

Rutter M, Quinton D. Parental psychiatric disorder: effect on children. Psychol Med 1984;14: 853-80

Billing AG, Moos RH. Comparisons of children of depressed and non-depressed parents: a socialenvironmental perspective. f A hnorm Child Psychol 1983:11:463-86.
3 Rutter M. Family and school influences on cognitive development. I Child Psychol Psychiatr. $1985 ; 26: 683-704$

4 Brown G, Harris T. Social ongins of depression. London: Tavistock, 1978

5 Moss P, Plewis I. Mental distress in mothers of pre-school children in inner London. Psychol Med $1977 ; 7: 641-52$

6. Richman N, Stevenson JE, Graham PJ. Prevalence of behavioural problems in three year old children: an epidemiological study in a London borough. $f$ Child Psychol Psychiatry children: an

7 Mills M, Puckering C, Pound A, Cox AD. What is it about depressed mothers that influences their children's functioning? In: Stevenson J, ed. Recent research in developmental psychopatholog Oxford: Pergamon (in press). (Joumal of Child Psychology and Psychiatry monograph, supplement 4.

8 Rutter $M$, Cox A, Tupling C, Berger M, Yule WL. Attainment and adjustment in two geographical areas. I. The prevalence of psychiatric disorder. Brf Psychiatry 1975;126:493-509.

Rutter M, Yule B, Quinton D, Rowlands D, Yule W, Berger M. Attainment and adjustment in two geographical areas. II. Some factors accounting for area differences. Br $\mathcal{J}$ Psychiatny 1975;126:520-33

10 Cooper SF, Leach C, Stover D, Tonge WL. The children of psychiatric patients: clinical findings. Br J Psychiatn 1977;131:514-22.

11 Brockington IF, Kumar R. Motherhood and mental illness. London: Academic Press, 1982.

12 Weissman MM, Paykel ES, Klerman GL. The depressed woman as a mother. Soc Psychiat 1972;7:98-108

13 Livingood $A B$, Daen $P$, Smith $B$. The depressed mother as a source of stimulation for her infant J Clin Psychol 1983;39:369-75.

14 Herbert M. Sluckin W. Sluckin A. Mother-to-infant "bonding." 7 Child Psychol Psychiatn. 1982:23:205-21

15 Kumar R, Robson KM. A prospective study of emotional disorders in childbearing women. $\mathrm{Br} f$ Psychialn 1984:144:35.47.

16 Chess S. Thomas A, Birch HG, Hertzig M. Implications of a longitudinal study of child development for child psychiatry. Am $\mathcal{F}$ Psychiatry 1960;117:434-41.

17 Rutter $M$, Birch HG, Thomas A, Chess S. Temperamental characteristics in infancy and the later development of behavioural disorders. Br $\mathcal{F}$ Psychiatry 1964;110:651-61

18 Uddenberg N, Englesson I. Prognosis of post partum mental disturbance. Acta Psychiatr Scand 1978:58:201-12

19 Ghodsian M, Zaiicek E, Wolkind S. A longitudinal study of maternal depression and child behaviour problems. F Child Psychol Psychiatn 1984:25:91-109.

20 W'rate RM, Roonev AC, Thomas PF, Cox JL. Postnatal depression and child development: a three year follow up study. Br F Psychiatry 1985:146:622-7.

21 McCarthy D. Manual for the McCarths scales of children's abilutes. New York: Psychological Corporation, 1972

2 Carev $W_{B}$, MCDevitt SC. Revision of the infant temperament questionnaire Pediutrics $1978: 61: 735-9$

23 Richman N, Graham PJ. A behavioural screening questionnaire for use with three year old children: preliminary findings. I Child Psychol Psychiatry 1971:12:5-33.

24 Goldberg D, Eastwood MR, Kedward HB, Shepherd M. A standardised psychiatric interview for Goldberg D. Eastwond MR, Kedward HB, Shepherd M. A standar
use in community surveys. Br f Prev Soc Med 1970;24:18-23.

25 Prechtl HFR. Neurological sequelac of prenatal and perinatal complications, Br Med 7 $1967 ;$ ii: $763-7$

26 Davie R, Butler R, Goldstein H. From birth to seven: a report on the National Child Development itudy: London: Longman, 1972

27 Madge N, lizard J. Intelligence. In: Rutter M, ed. Scientific foundations of developmental psychiatry. London: Heinemann, 1980:245-65.

28 Pitt B. Atypical depression following childbirth. Br f Psychiatry 1968;114:1325-35.

\title{
Treatment of first episodes of acute anal fissure: prospective randomised study of lignocaine ointment versus hydrocortisone ointment or warm sitz baths plus bran
}

\author{
STEEN LINDKÆR JENSEN
}

\begin{abstract}
One hundred and three patients with an acute first episode of posterior anal fissure were randomised to receive a three week trial of lignocaine ointment $(n=33)$ versus hydrocortisone ointment $(n=35)$ or warm sitz baths combined with an intake of unprocessed bran $(n=35)$. Seven patients were withdrawn owing to failure to adhere to the trial protocol. After one and two weeks of treatment symptomatic relief was significantly better among
\end{abstract}

General Practice and Surgical Clinic, Lægehuset, Rønnebær Allé, Elsinore, and Department of Surgical Gastroenterology C, Rigshospitalet, University of Copenhagen, Denmark

STEEN LINDKÆR JENSEN, MD, DRMED, senior registrar

Correspondence to: Dr Steen Lindkær Jensen, Department of Surgical Gastroenterology C2122, Rigshospitalet, Blegdamsvej 9, DK-2100 Copenhagen 0, Denmark. patients treated with sitz baths and bran than among patients treated with lignocaine ointment or hydrocortisone ointment. After three weeks there was no difference in symptomatic relief among the three groups. Patients treated with lignocaine, however, had significantly fewer healed fissures $(60 \%)$ than patients treated with hydrocortisone $(82 \cdot 4 \%)$ or warm sitz baths and bran $(87 \%)$.

In this study warm sitz baths plus an intake of unprocessed bran came out as the treatment of choice for an acute first episode of posterior anal fissure. This treatment is cheap, has no potential serious side effects, and brings the best and quickest relief of symptoms.

\section{Introduction}

Treatment of an acute anal fissure aims at relieving pain and reflex spasm often caused by a hard stool. Decisions on treatment seem to be based on clinical experience rather than on clinical or scientific 
premises, since results from randomised series are lacking. Clinical textbooks teach that an acute anal fissure can be treated by simple measures such as warm sitz baths and stool softeners. ${ }^{~}$ Nevertheless, despite this and the spontaneous healing that occurs with time many expensive suppositories, ointments, and creams have been developed for the condition. All are widely used but their efficacy remains unproved.

I report a controlled prospective randomised study comparing the short term effects of lignocaine ointment, hydrocortisone ointment, and a combination of warm sitz baths and unprocessed bran intake in patients with a first episode of acute anal fissure.

\section{Patients and methods}

Between June 1980 and December 1983 patients seen within three weeks of onset of a first episode of anal pain and spasm caused by a deep ulcer (fissure) in the anal lining posteriorly between the level of the anal valves and the anal orifice entered the study. The study excluded pregnant women; patients with concomitant inflammatory bowel disease; patients with signs of a chronic fissure (that is, one in which the fibres of the internal sphincter were visible in the base and with fibrous induration of the lateral edges); patients with sentinel piles or hypertrophied anal papillas, or both; and patients with only an abrasion of the skin of the anal region. Patients whose fissures were localised anteriorly or laterally were also excluded.

\section{STUDY DESIGN}

At the initial clinic visit a standard medical history was obtained and the diagnosis of an acute posterior anal fissure made by inspection and palpation. Complete digital examination was often impossible at the initial visit owing to severe pain and spasm. Anosigmoidoscopy also could not be performed at the initial examination but was done later to rule out other lesions or diseases of the anorectum.

The patients were assigned at random to the following three treatment groups.

Lignocaine ointment-Patients were instructed to insert $2 \%$ lignocaine ointment into the anal canal in the morning and at bedtime and after each stool throughout the day. Of 33 patients assigned to this treatment, three failed to attend for follow up.

Hydrocortisone ointment-Patients were instructed to insert $2 \%$ hydrocortisone ointment into the anal canal in the morning and at bedtime and after each stool throughout the day. Of 35 patients assigned to this treatment, one failed to follow the study protocol.

Patients receiving either lignocaine or hydrocortisone ointment were warned that application to the perianal skin only would be of no help.

Warm sitz baths plus unprocessed bran-Patients were instructed to take their usual diet plus $10 \mathrm{~g}$ of unprocessed bran in the morning and $10 \mathrm{~g}$ at bedtime and to use a warm sitz bath for 15 minutes at these times and (if possible) after each stool throughout the day. Patients were told to use a bathtub or simply a bowl filled with hot water $\left(40^{\circ} \mathrm{C}\right)$. Thirty five patients were assigned to this treatment; three failed to follow the study protocol.

Patients were examined one, two, and three weeks after beginning treatment and had their clinical state assessed by an independent observer who did not know which treatment had been given. Symptomatic responses were assessed in terms of relief of symptoms and sphincter spasm. Subjective improvement was assessed by all patients using a 10 point scale at each examination. Absence of symptoms was scored as zero; moderate and severe pain were scored as 5 and 10 respectively.

\section{STATISTICAL ANAL YSIS}

Statistical evaluation was by the $\chi^{2}$ test or Mann-Whitney U test. No power estimations were performed. $p$ Values less than 0.05 were considered significant.

\section{COMPLIANCE}

Consumption of bran, use of ointments, number of warm sitz baths, and daily dietary intakes were recorded on diary cards. In the group treated with bran the patients took $90.6 \%$ of their prescribed bran and $88.8 \%$ of their warm sitz baths. In the groups treated with ointments the patients given lignocaine used $96.2 \%$ of the prescribed ointment and the patients given hydrocortisone $91.4 \%$. Daily dietary intake was equal in the three groups. Five patients (two in the lignocaine treated group, two in the hydrocortisone treated group, one treated with sitz baths and bran) took laxatives $\stackrel{\text { W }}{-}$ periodically during the study. The amount of laxatives used was small, $\underset{Z}{\lessgtr}$ however, and was not thought to have any effect on the results.

\section{Results}

Ninety six patients completed the study. Table I compares the three $\mathbb{D}$ treatment groups with regard to age, sex, duration of symptoms, number of patients practising anal intercourse, and number of chronic users of patients

Table II shows the presenting symptoms of patients in the study. Pain during and after defecation was the most common problem followed by bleeding and constipation.

TABLE I-Characteristics of study population

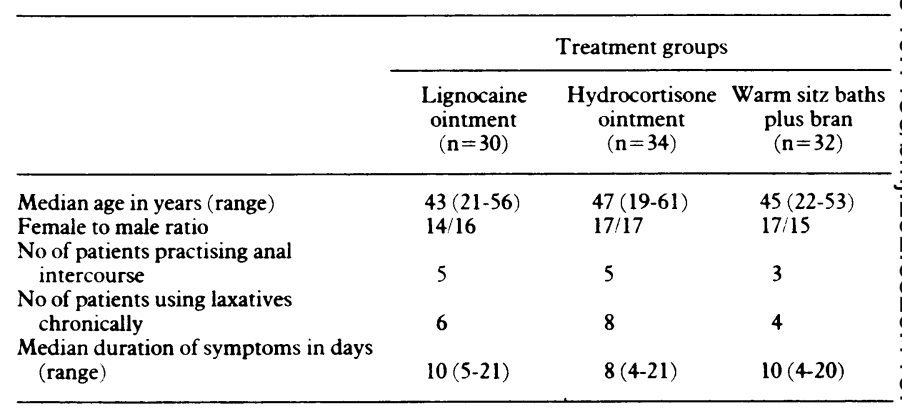

(and

TABLE II-Presenting symptoms. Figures are numbers of patients (percentages in 3 parentheses)

\begin{tabular}{lccccc}
\hline Symptoms & \multicolumn{3}{c}{ Treatment groups } & \\
\cline { 2 - 4 } & $\begin{array}{c}\text { Lignocaine } \\
\text { ointment } \\
(\mathbf{n}=30)\end{array}$ & $\begin{array}{c}\text { Hydrocortisone } \\
\text { ointment } \\
(\mathbf{n}=34)\end{array}$ & $\begin{array}{c}\text { Warm sitz baths } \\
\text { plus bran } \\
(\mathbf{n}=32)\end{array}$ & $\begin{array}{c}\text { Total } \\
(\mathbf{n}=96)\end{array}$ \\
\hline Pain & $30(100 \cdot 0)$ & $34(100 \cdot 0)$ & $32(100 \cdot 0)$ & $96(100 \cdot 0)$ \\
Bleeding & $24(80 \cdot 0)$ & $26(76 \cdot 5)$ & $24(75 \cdot 0)$ & $74(77 \cdot 1)$ \\
Constipation & $20(66 \cdot 6)$ & $24(70 \cdot 6)$ & $21(65 \cdot 6)$ & $65(67 \cdot 7)$ \\
Pruritus ani & $10(33 \cdot 3)$ & $14(41 \cdot 2)$ & $12(37 \cdot 5)$ & $36(37 \cdot 5)$ \\
Soiling & $6(20 \cdot 0)$ & $5(14 \cdot 7)$ & $6(18 \cdot 8)$ & $17(17 \cdot 7)$ \\
Diarrhoea & $3(10 \cdot 0)$ & $3(8 \cdot 8)$ & $2(6 \cdot 3)$ & 8 & $(8 \cdot 3)$ \\
\hline
\end{tabular}

In eight men and two women the causative factor in development of the fissure was thought to be trauma to the anal canal during anal intercourse, whereas in 65 patients passage of a hard faecal bolus was the probable cause. In eight patients with a history of chronic misuse of laxatives the fissure 3 developed during a period of severe diarrhoea, and in 13 patients no causative factor could be identified.

At the first visit 94 of the patients were judged to have severe sphincter spasm at examination and two mild to moderate spasm.

Relief of symptoms-The figure plots the symptomatic profiles during the $N$ three weeks of treatment. There was a progressive decrease in discomfort in $\square$ each treatment group during each week. During the first two weeks the $\frac{D}{2}$ decrease in symptom score was significantly greater in patients treated with warm sitz baths plus bran $(\mathrm{p}<0.01$ and $\mathrm{p}<0.05$ respectively) than in patients $\widetilde{O}$ using lignocaine or hydrocortisone ointment. There was no significant $N$ difference in relief of symptoms between the two groups treated with ointments. After three weeks symptomatic relief was the same regardless of the treatment regimen.

Healing rates-Table III shows the cumulative healing rates of first episode acute posterior anal fissures in relation to the three treatment ${ }^{-}$ regimens tested. At one and two weeks the healing rate was significantly $\underset{T}{T}$ better among patients treated with warm sitz baths than among those treated $\frac{}{\mathbb{D}}$ with lignocaine ointment $(p<0.01)$ or hydrocortisone ointment $(p<0.05)$. After three weeks there was no difference in healing rate between patients treated with sitz baths and bran and those treated with hydrocortisone $\sigma$ ointment, whereas the healing rate in patients treated with lignocaine ointment was significantly lower than in the sitz baths and hydrocortisoneo treated groups $(\mathrm{p}<0.025$ and $\mathrm{p}<0.05$ respectively). Three weeks after beginning treatment $74(77 \cdot 1 \%)$ of the 96 patients had healed. Of the remaining patients, 16 were submitted to lateral subcutaneous internal 


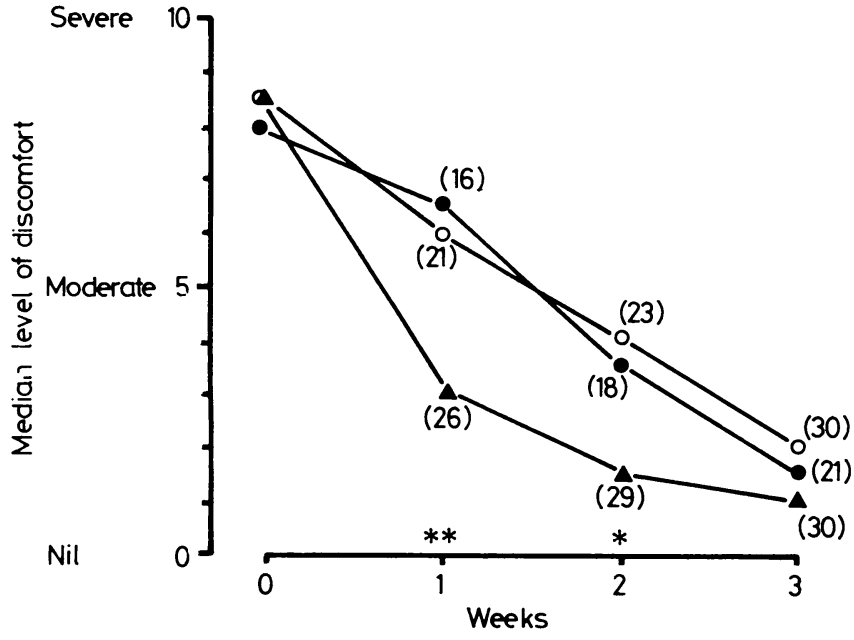

Median levels of discomfort in patients with first episode of posterior acute anal fissures before and during treatment with lignocaine ointment $(0, n=30)$, hydrocortisone ointment $(\mathrm{O}, \mathrm{n}=34)$, or warm sitz baths plus intake of unprocessed bran $(\boldsymbol{\Lambda}, \mathbf{n}=32)$. Figures in parentheses are numbers of patients showing improvement in score each week.

${ }^{\star} \mathrm{p}<0.05 .{ }^{\star \star} \mathrm{p}<0.01$.

TABLE III-Cumulative healing rates of first episode acute anal fissures in relation to treatment regimens. Figures are numbers of patients with healed fissures (percentages in parentheses)

\begin{tabular}{lrrr}
\hline & 1st Week & 2nd Week & 3rd Week \\
\hline Lignocaine ointment $(n=30)$ & $6(20 \cdot 0)$ & $12(40 \cdot 0)$ & $18(60 \cdot 0)$ \\
Hydrocortisone ointment $(n=34)$ & $11(32 \cdot 4)$ & $22(64 \cdot 7)$ & $28(82 \cdot 4)$ \\
Warm sitz baths plus bran $(n=32)$ & $15(46 \cdot 9)$ & $24(75 \cdot 0)$ & $28(87 \cdot 5)$ \\
\hline
\end{tabular}

sphincterotomy for persistent symptoms, ${ }^{2}$ whereas six patients were symptom free and were.not treated further. Biopsy samples from these six patients showed no signs of malignancy.

Side effects-One homosexual patient treated with hydrocortisone ointment developed extensive herpetic lesions locally. Thirteen of the 32 patients $(40.6 \%)$ treated with unprocessed bran felt distended and suffered various degrees of flatulence when they took their bran. None stopped taking the bran on this account. No side effects occurred in patients treated with lignocaine ointment.

\section{Discussion}

This study shows that in patients with a first episode of acute posterior anal fissure simple measures such as warm sitz baths combined with a dietary intake of unprocessed bran may relieve symptoms significantly better than the application of lignocaine or hydrocortisone ointment to the anal canal.

Our patients were characterised by their homogeneity. All had first episode anal fissures localised posteriorly without any sign of a chronic fissure and with a very short duration of symptoms. This stands in contrast with most other published series, ${ }^{3.5}$ which were characterised by the complex mix of patients studied-varying in localisation of the fissures, durations of symptoms, number of patients with recurrent fissures, and number with signs of chronic fissures. This important difference must be kept in mind when evaluating our results.

Many acute fissures are said to heal spontaneously within one to two weeks. ${ }^{6}$ Nevertheless, these fissures cause much suffering and patients will often seek help. Of the several treatment regimens available, however, which is the most favourable is a subject of controversy. ${ }^{16}$
Anaesthetic ointments have been used with varying success. ${ }^{3.5}$ Thus in a randomised study by Gough and Lewis application of $2 \%$ lignocaine healed posterior fissures in $43.6 \%$ of patients within one month. ${ }^{3}$ These patients had a mixture of different fissures; the mean duration of symptoms was 21.1 months (range 0.5 to 120 months) and $48 \cdot 7 \%$ of the patients also had a sentinel pile. In our study lignocaine ointment showed no convincing effect on relief of symptoms or on the healing rate of the fissures. Furthermore, a perianal dermatitis may occur with the use of anaesthetic ointments. ${ }^{\prime}$ Hence there seems little to justify treating acute anal fissures with these ointments.

Several other different ointments and suppositories exist for the treatment of different minor anal diseases, including anal fissures. They share in common different proportions of several combinations of anaesthetics, astringents, and anti-inflammatory agents (usually hydrocortisone) in a host of bases and preservatives. For the ointment to be of any help it must be inserted into the anal canal; application only to the perianal region would be of no use. Most patients find this procedure distasteful as well as uncomfortable. Though these ointments and suppositories are prescribed by doctors all over the world, ' so far as we know their effectiveness has never been tested by a randomised trial. We chose hydrocortisone as a model of this treatment regimen and it did not contribute successfully to the conservative management of our patients. Relief of symptoms was significantly poorer than that obtained with warm sitz baths and dietary bran. Furthermore, one homosexual man developed extensive local spread of a previously undiagnosed anal herpes.' Our findings do not seem to show any beneficial effect of ointments containing hydrocortisone in acute anal fissures, though the healing rate three weeks after treatment was the same as with warm sitz baths plus bran.

Other conservative treatment regimens such as use of an anal dilator with or without an anaesthetic ointment or injecting long acting anaesthetics, ${ }^{348}$ also share in a lack of prospective randomised studies of their effect on entirely acute anal fissures. Nowadays, however, they probably have no place in treatment owing to bad results, unpleasant methods of application, and serious side effects. ${ }^{6}$

From our study avoiding constipation and sphincter spasm seem to be important items of conservative treatment of acute anal fissures. Warm sitz baths are believed to relieve sphincter spasm and unprocessed bran to produce large bulky stools resulting in a physiological dilatation of the anal sphincter, thereby healing the fissures. Instead of unprocessed bran, which many patients may find unpalatable, bran compressed as tablets may be used. ${ }^{9}$ We chose unprocessed bran because it is the cheapest substitute for cellulose fibre missing from the modern diet in Denmark.

Our findings show plainly that most patients with a first episode acute posterior anal fissure can be treated successfully with warm sitz baths combined with unprocessed bran, thereby avoiding the frequently used anaesthetic or anti-inflammatory ointments. These agents are less effective, more expensive, and have unpleasant side effects.

\section{References}

1 Goldberg SM, Gordon PH, Nivatvongs S. Essentials of anorectal surgery. Philadelphia, Toronto: Lippincott, 1980

2 Jensen SL, Lund F, Nielsen OV, Tange G. Lateral subcutaneous internal sphincterotomy versus anal dilatation in the treatment of fissure in ano in outpatients: a prospective randomised study. BrMed f 1984;289:528-30.

3 Gough MJ, Lewis A. The conservative treatment of fissure-in-ano. Brf Surg 1983;70:175-6.

4 Lock MR, Thomson JPS. Fissure-in-ano: the initial management and prognosis. Br $\mathcal{f}$ Surg 1977;64:355-8.

5 Fries B, Rietz KA. Treatment of fissure in ano. Acta Chir Scand 1964;128:312-5.

6 Goligher JC. Surgerv of anus, rectum, and colon. 4th ed. London: Ballière, Tindall, 1980.

6 Goligher JC. Surgery of anus, rectum, and colon. 4th ed. London: Ballière, Tindall, 1980.

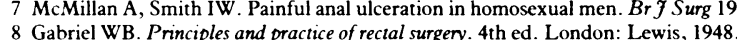

8 Gabriel WB. Principles and practice of rectal surgery. 4th ed. London: Lewis, 1948.
9 Tayler I, Duthie HL. Bran tablets and diverticular disease. Br Med F 1976;i:988-90.

(Accepted 27 March 1986) 